\title{
Fischer's Plants in folk beliefs and customs: a previously unknown contribution to the ethnobotany of the Polish-Lithuanian- Belarusian borderland
}

Monika Kujawska ${ }^{1 *}$, Piotr Klepacki ${ }^{2}$ and Łukasz Łuczaj $^{3}$

\begin{abstract}
Background: Historical ethnobotanical studies are useful starting points for further diachronic analysis. The aim of this contribution is to present archival data from the Polish-Lithuanian-Belarusian borderland, which were collected by Adam Fischer, a Polish ethnographer from Lviv, in the 1930s. These data were originally gathered for publication in the first part of the Lexicon of Slavic beliefs and customs, dedicated to plant uses in traditional Slavonic culture. It was intended to be a joint international enterprise, but was never actually fulfilled.

Methods: In this article we used information from historical Lithuania (the Great Duchy of Lithuania), nowadays a border region between Poland, Lithuania and Belarus. We applied cultural importance indices such as Use Value, Relative Importance value and Sørensen similarity coefficient, in order to compare our data with a western Ukraine data set from the same research framework.

Results: In total, 153 plant taxa were registered as used in peasant culture in the Polish-Lithuanian-Belarusian borderland in the 1930s. The species which achieved the highest Use Values were: Calendula officinalis, Cyanus segetum, Helichrysum arenarium, Betula sp., Prunella vulgaris, and Nuphar lutea or Lilium sp. The most salient use categories were medicinal, followed by food and home garden plants. The overall similarity to plants recorded in western Ukraine within the same project of Fischer's is quite low (46\%), which may be explained by the partly different flora found in the regions, and a cultural discontinuity, revealed by the difference in species with the highest UV. Moreover, the field collaborators were different in the two regions and may have paid attention to different cultural spheres of use.
\end{abstract}

Conclusions: The presented ethnobotanical data are a valuable contribution to the ethnobotany of Eastern Europe as a whole. In particular, the presented list of plants may be a rich source for future studies on the ethnobotany of the Polish diaspora in Lithuania, and diachronic studies in north-east Poland and Belarus.

Keywords: Historical ethnobotany, Lithuania, Poland, Belarus, Medicinal plants

\footnotetext{
*Correspondence: monika.kujawska@uni.lodz.pl

'Institute of Ethnology and Cultural Anthropology, University of Łódź,

Lindleya 3/5, 90-131 Łódź, Poland

Full list of author information is available at the end of the article
} 


\section{Background}

Ethnobotanical studies concerning Lithuania and Belarus The archival ethnobotanical data presented in this paper correspond to a historically and culturally complex region. In medieval times it formed part of the Great Duchy of Lithuania. Due to political influences and cultural exchange, the former territory of the Grand Duchy of Lithuania was partly polonized. Between the $16^{\text {th }}$ and $18^{\text {th }}$ centuries Poland and Lithuania formed a kind of commonwealth under the same ruler [1]. Currently the areas from which our information comes belong to the north-eastern outskirts of Poland, Belarus and Lithuania.

In contrast to some other Eastern European countries such as Poland, Hungary or Estonia, there have been very few publications concerning the ethnobotany of Lithuania [2]. This is the case, strangely, both for Lithuanians and for the large Polish population inhabiting the territory of present Lithuania [3]. Some published articles only concern the names of plants and their symbolic meaning in Lithuanian folk culture [4-6]. Contemporary researchers attempt to explain this phenomenon by analysing historical processes, especially the modernization process of the young Lithuanian state from the beginning of the $20^{\text {th }}$ century onwards. In this intellectual context there was little interest in recording ethnobotanical or, especially, ethnomedical knowledge. Instead, ethnologists focused on the poetics of folklore, and within the framework of folk medicine the main objects of interest were verbal formulas, associated with charm healing. Such representation of folk medicine, in conjunction with the ritual use of herbs, was the cause of a stereotypical perception of traditional medicine as an irrational practice [7]. Jan Balys (1909-2011), the prominent Lithuanian ethnologist, deepened the chasm of misunderstanding, claiming that "the largest part of folk medicine is based on similarities (analogies) and has no therapeutic relevance" [8]. Moreover, the territory of the Grand Duchy of Lithuania was for centuries within the radius of activity of Polish culture. However, the $19^{\text {th }}$ and $20^{\text {th }}$ century folklorists concentrated exclusively on Slavic peasants inhabiting the former territory of the Polish-Lithuanian Commonwealth. Even from the Polish language publications in Lithuania, however, we can only list the inventory of medicinal plants sold in the annual Midsummer Herbal Market of Vilnius collected in 1920s by the Polish pharmacist and botanist Jan Muszyński [9]. The author counted more than 100 species, and provided their botanical names, uses and folk names. Some information on plant uses contains vague references to Lithuania in old Polish herbals and economic books $[10,11]$. However, the Duchy of Lithuania was huge, hence they could refer to the present Belarus (Polesia), or parts of NE Poland (Podlasie). Rostafinski's questionnaire of 1883 did not provide any ethnobotanical data either, as the volume of letters from Lithuania is missing from the preserved collection of responses to his questionnaire. Thus the information gathered by Fischer contains the most diverse collection of data, especially on the ethnobotany of the Polish ethnic group in the Vilnius area, the present border region between Belarus and Lithuania.

The ethnobotany of Belarus is much better studied than that of Lithuania, but still insufficiently, taking into account its living tradition of plant use. Kazimierz Moszyński, as far back as 1914, wrote that Polesia (present Belarus) was the most attractive Polish territory (in the sense of the former Kingdom of Poland) from the ethnographic point of view [12]. In his book he included several folk botanical domains, such as food, medicinal and home garden plants. However several other studies are worthy of mention prior to his research, especially from the Grodno region, such as those of the Polish writer Eliza Orzeszkowa [13, 14]. Her lists of plants were based on in-depth fieldworks conducted over several springsummer seasons in the 1880s. Orzeszkowa's research was followed in the Grodno area by another female pioneer of ethnobotany, Zośka Wieras. Although born in Ukraine and of Polish-Lithuanian descent (she was also fluent in Russian and Ukrainian), she devoted herself to studying Belarusian folklore. In 1924 she published a Belarusian-Polish-Russian-Latin dictionary [15], which contains the names of a few hundred species of plants, based on her own field data and other sources. Polish ethnographer Michal Fedorowski documented the folk medicine of the $19^{\text {th }}$ century in the former eastern borderlands of the Polish Republic (Belarus today). His Belarusian folks...(1897) contains an entire chapter dedicated to plants used in folk therapies [16]. The information was supported by herbal specimens stored in the Warsaw University Botanical Garden [17]. Other minor works concerning the ethnobotany of Belarus were listed in the work by Łuczaj et al. [18]. These authors compared various materials concerning the use of wild foods in Belarus. These were mainly the unpublished questionnaires of Józef Rostafiński from 1883, as well as a handwritten monograph of Michał Fedorowski from the same period [16], as well as contemporary field work data by some of the co-authors of the paper. The contemporary studies dedicated to wild plants used as food, medicine and for animal wellbeing were performed recently in Liubań district, Minsk region by Sǒukand and collegues [19].

\section{The contribution of Adam Fischer: plants in folk beliefs and customs}

Adam Robert Fischer (1889-1943) was a Polish philologist, folklorist and ethnographer. From 1924 onwards he was a professor at the Department of Ethnography at the Jan Kazimierz University in Lwów (now Lviv) [20]. 
Fischer dedicated most of his life to the development of the Polish Ethnological Society and he also spent 33 years working as the editor-in-chief of $L u d$ - the oldest Polish ethnological journal [21]. The legacy of Professor Adam Fischer contains a rich collection of articles, books and unpublished materials, which is now owned by the Polish Ethnological Society. It was transported from Lviv to Wrocław after World War II by the professor's family [22]. The largest part of Fischer's ethnobotanical material was the result of his taking part in an international project called the Lexicon of Slavic beliefs and customs. The idea for this work arose during the I Congress of Slavic Philologists in Prague in 1929. Its first part was to be focused on plants in folk beliefs and customs. In order to accomplish this task, five editors were appointed from five Slavic countries: Christo Vakarelski from Sofia, Veselin Čajkanović from Belgrade, Karel Chotek from Prague, Adam Fischer from Lwów (now Lviv) and Dmitry Konstantinovich Zelenin from Leningrad (now Saint Petersburg). The mastermind of this enterprise was Edmund Schneeweis from Prague [23]. The Lexicon was to be published by the Walter de Gruyter editorial house. The progress of compiling the field work has already been described in our previous contribution [24]. Here we merely repeat the research topics that were investigated during the field campaign. They were published in the form of a questionnaire in 1929 [25] and again in 1930 [26]:

1) Local plant names and possible etymologies

2) Practical application and use of plants in everyday life, such as: food, construction material, cloths, dyeing agents, medicines and poisons

3) Plants with special magical powers, plants in love lore, bestowed with extraordinary virtues enabling the user to ascend into the air or to become invisible

4) Plants with symbolic significance in rituals and ceremonies, such as weddings, funerals and "chodzenie z maikiem" [spring custom of walking around a village with green branches, visiting households and singing songs by peasant youth]

5) Plants as decorative motifs present in houses, on cutlery, clothing, embroidery, cutouts and Easter eggs

6) Toys made from plants, e.g. cockerels, pipes, ropes; caps made of rushes, poppers made of elder, necklaces from rowan, fans, straws etc.

7) Plants in stories and folk songs

In addition to this questionnaire, Fischer enclosed an alphabetical list of 260 plant species according to Polish common names, with Latin names in brackets, which could serve as a prompt for field collaborators. In the archive of the Polish Ethnological Society, stored letters from field collaborators may be found, observing that the list could not have been very productive in the course of collecting materials, as peasants did not know the Polish common names for plants in most cases (Wincenty Bandrowski, Archiwum PTL, sygn. 356). The field information was coupled with voucher specimens, which were identified in the Institute of Botany at Jan Kazimierz University and then returned to field workers. Therefore, no specimens were preserved at the Polish Ethnological Society.

Fischer was interested in the whole area of pre-World War II Poland, which also includes present western Ukraine and parts of Belarus and Lithuania. He did not manage to publish the results of his research. The aim of this contribution is to describe and analyse a portion of the data set collected for the editing of the Lexicon, which corresponds to the historical medieval Lithuania. Nowadays this is NE Poland (Suwałki region), Belarus (Oszmiana area, Polesia region) and Lithuania (Kowno, Troki, etc.) (Fig. 1, Table 1). We also aim to compare this material with the data set from western Ukraine coming from the same period and conducted within the same research framework [24], with regard to the overall composition of species, and medicinal plants in particular.

\section{Methods}

Study area

The current borderland between Poland, Belarus and Lithuania used to be part of the Grand Duchy of Lithuania, a European state from the $13^{\text {th }}$ century until 1795, founded by the Lithuanian Baltic tribe. The Grand Duchy, in its expansion, included part of the territories of the present day Belarus, Poland, Ukraine and Russia. The Union of Krewa in 1385 brought two major changes for the Grand Duchy - conversion to Catholicism (it was previously a pagan state) and a dynastic union with the Kingdom of Poland. From 1569 onwards, the union was converted into a Polish-Lithuanian Commonwealth, and Polish became the official language [27]. It was a multi-ethnic, multi-language and multi-religious state. Two macro ethnic groups lived there, the Balts (Lithuanians) and the Slavs (Belarus, Poles, Russians, Ukrainians), as well as smaller groups, such as Jews, Gipsies, Tatars and Karaites [3]. The latter were of Turkic origin, preserving their own language and religion, originating from Judaism [28]. Different religious minorities were encouraged to settle in the Grand Duchy due to the confessional freedom that ruled in the state. Opportunities to practice traditional forms of healing were also probably more favorable in Lithuania in comparison with Western Europe, or Poland, where persecution for witchcraft was widespread [7]. The Russian invasion in 1792 led to the partition of The Duchy between 


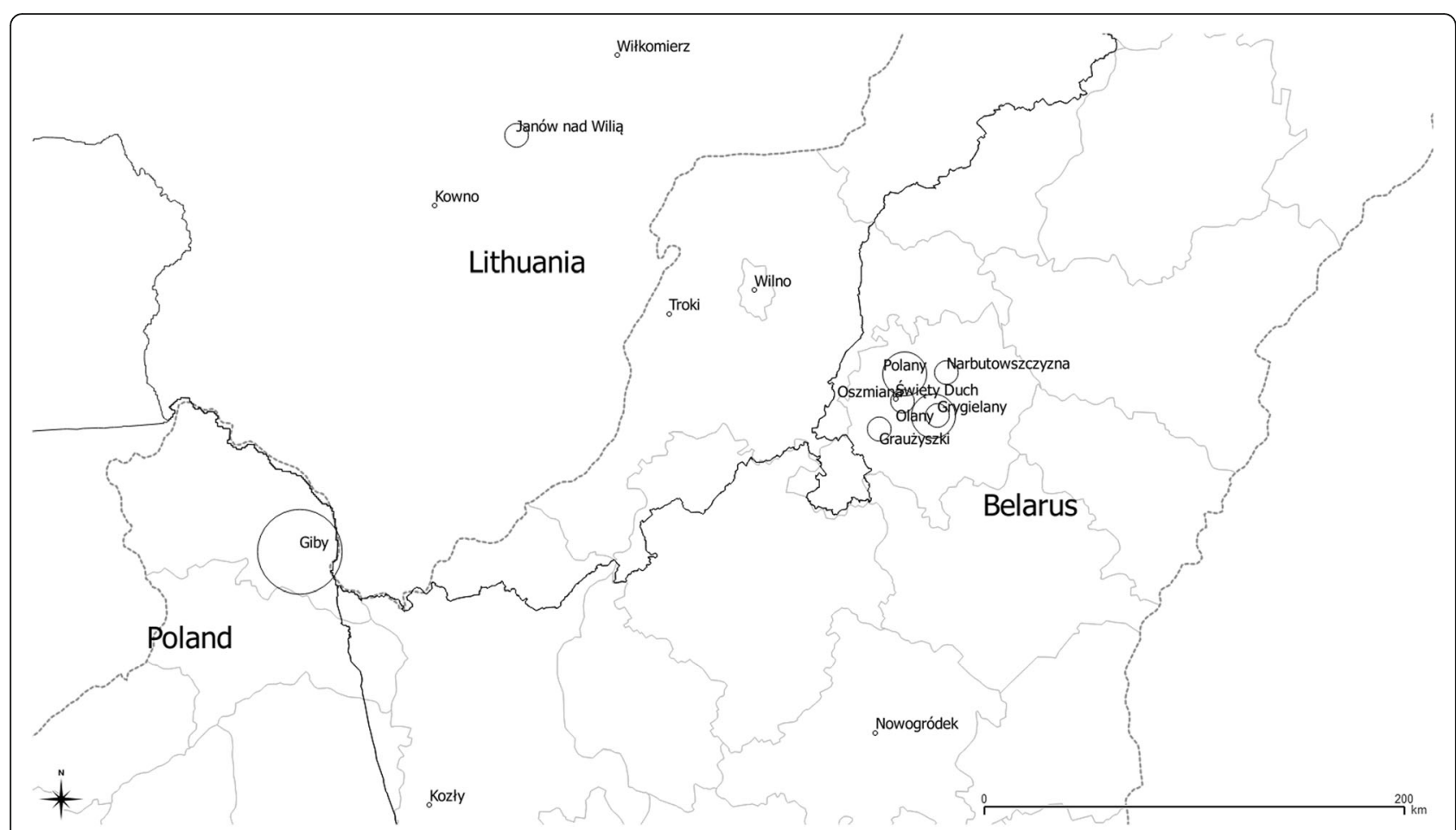

Fig. 1 Distribution of the study localities in the 1930s in Polish-Lithuanian-Belarusian borderland. The dotted line marks the pre-1939 border of Poland. Circles indicate a size of the dataset from each locality

the Russian Empire and Prussia in 1795. After World War I, the Vilnius area and part of Belarus were incorporated into the newly formed Polish Republic as an outcome of the treaty of Riga, local wars and, in case of Vilnius, due to a ruse [1].

Table 1 Historical and current names of villages and areas studied by Adam Fischer's field collaborators

\begin{tabular}{lll}
\hline Locality & Contemporarty name & Country \\
\hline Giby & Giby & Poland \\
Grygielany & Гиргеляны & Belarus \\
Janów nad Wilią & Jonava & Lithuania \\
Kowno (and Kowno area) & Kaunas & Lithuania \\
Kozły & Козлы & Belarus \\
Narbutowszczyzna & Нарбуты & Belarus \\
Nowogródek & Навагрудак & Belarus \\
Graużyszki area & Гравжишки & Belarus \\
Wilno area & Vilnius & Lithuania \\
Wiłkomierz area & Чктегgè & Lithuania \\
Olany & Оляны & Belarus \\
Oszmiana & Ашмяны & Belarus \\
Polany & Поляны & Belarus \\
Święty Duch & Будзёнаўка & Belarus \\
Troki & Тгакаі & Lithuania \\
\hline
\end{tabular}

\section{Data collection and botanical identification}

Information from the described region was collected during the period between 1929-1935 by several of Adam Fischer's field collaborators. The field research was conducted in the Polish language among Polish speaking peasants from these territories. We know two field collaborators by name, and the localities in which they conducted their field research (Table 2). However, the number of informants they included in the study remains unknown. Additionally, data came from a manuscript stored in Ossolineum (National Ossoliński Institute) in Wroclaw [29]. These data were mainly from the Janów nad Wilią, Kowno, Troki (nowadays Lithuania).

The data set from the Polish-Lithuanian-Belarusian borderland comprises of 283 filecards. Sometimes different cultural uses were lumped together on a single card and sometimes they were split (Fig. 2).

Table 2 Adam Fischer's field collaborators in the historic Lithuania

\begin{tabular}{llll}
\hline Name of field collaborator & Province & County & Locality \\
\hline Koczorówna Zofia & białostockie & Suwałki & Giby \\
Koczorówna Zofia & wileńskie & Oszmiana & Narbutowszczyzna \\
Perls H. & wileńskie & Oszmiana & Grygielany \\
Perls H. & wileńskie & Oszmiana & Olany \\
Perls H. & wileńskie & Oszmiana & Polany \\
\hline
\end{tabular}




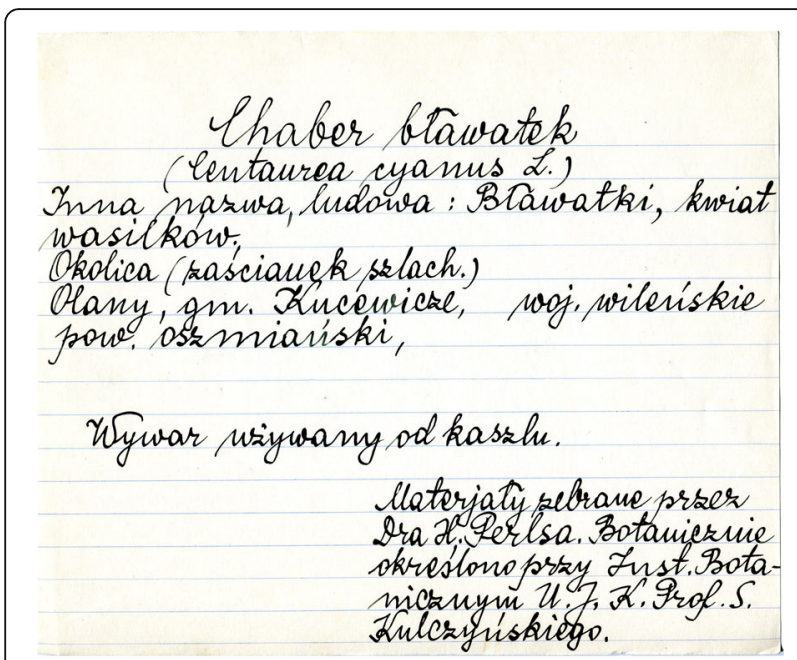

Fig. 2 An exemplar filecard with information on plant names, uses and botanical identification from the Vilnius region. [Exact translation: Chaber bławatek (Polish vernacular name), followed by Latin name, folk name: bławatki, kwiat wasilików. Locality: Manor area, Olany, County: Oszmiana, Province: Wilno [Vilnius, now this part is in Belarus]. Use description: decoction used for cough. The information was gathered by $\mathrm{H}$. Perls and botanical material was identified in the Institute of Botany in the University of Jan Kazimierz in Lwów (now Lviv)]

Out of 153 plant taxa whose uses were registered for the study region, nearly all were identified in the Botanical Institute of the Jan Kazimierz University (JKU) in Lwów. However, we do not know if the information recorded in Łaguna's manuscript underwent botanical identification [29]. All Latin names provided by botanists from the JKU were verified according to the Plant List database [30]. Other Latin names were estimated by the authors, based on common Polish names and local names - as some of the filecards contained both, and some only one of them. The estimated names were then cross-checked with the Polish ethnobotanical and ethnographic literature described in the Background. Cases such as carrot, coriander, caraway and hemp were easy to solve. On several occasions, we were not sure of the correctness of identification, thus we put a question mark (?) next to it. Still, there were some filecards which contained only local names, such as mieniacy kwiat (shining flower), krowi kwiat (cow's flower) and polska korona (Polish crown), which we could not ascribe to any botanical species - hence these plants and their uses were not included in the analysis.

\section{Data analysis}

A database was used to perform a detailed description of the categories in which plants were used in the PolishLithuanian-Belarusian borderland in the 1930s. The material was divided into use categories such as: medicinal, food, household, ritual, veterinary and plants cultivated in home gardens. These were etic constructions, which enabled us to apply the Use Value index proposed by Prance et al. [31], and they were modified according to the requirements of archive data. Therefore, in our study each use of a plant taxon was counted as 1.0. Some species had different uses within one category - these were counted as separate uses. Then, for each taxon, we summed up the values corresponding to its uses.

In order to compare the composition of species used in the study region with the data set coming from western Ukraine and collected within the same piece of research, we used the Sørensen similarity coefficient. The formula is as follows: $\mathrm{Ss}=2 \mathrm{a} /(2 \mathrm{a}+\mathrm{b}+\mathrm{c})$, where $\mathrm{a}=$ number of shared species, $\mathrm{b}=$ number of exclusive species group $1, \mathrm{c}=$ number of exclusive species group 2 . The result is then multiplied by 100 , in order to express it as percentage.

The second index of cultural importance we applied was used for medicinal plants only. This was the Relative Importance (RI) value proposed by Bennett and Prance [32], designed to measure medicinal plant versatility. It takes into account two factors: the relative number of body systems (RelBS) treated with a given plant taxon and the relative number of pharmacological properties (RelPH) ascribed to the species. Therefore, this index was appropriate for our set of data, as the number of informants (which is normally required in importance indices) was missing.

\section{Results and discussion General findings}

In total, 153 plant taxa with 290 uses were still used, or only remembered, in peasant culture in the 1930s in the Polish-Lithuanian-Belarusian borderland (Table 3). The species which achieved the highest Use Value were: Calendula officinalis (5), Cyanus segetum (5), Helichrysum arenarium (5), Betula sp. (4), Prunella vulgaris (4), and Nuphar lutea or Lilium sp. (4). Compared to plants used in folk culture in western Ukraine during the same period of time, less species were used (153 and 179, respectively). The most versatile species in the two regions were totally different. According to our previous piece of research, the most versatile plant species in western Ukraine were: Achillea millefolium (7), Allium sativum (6), Vinca minor (6), Hypericum sp. (5), Juniperus communis (5) [24]. The overall similarity between the two regions is $46 \%$, according to the Sørensen similarity coefficient. This can be explained by the partly different flora found in both regions, and a cultural discontinuity revealed in the difference between species with the highest UV. Moreover, the field collaborators were different in the regions and may have paid attention to different cultural spheres of use. This last observation may explain to some extent the fact that, apart from medicinal plants, which were the most important semantic 
Table 3 List of plant species used in folk culture of Polish-Lithuanian-Belarusian borderland

\begin{tabular}{|c|c|c|c|c|}
\hline Species & Local name & Category of uses & Use value & $\begin{array}{l}\text { Relative } \\
\text { importance } \\
\text { value }\end{array}$ \\
\hline Achillea millefolium $\mathrm{L}$. & krwawnik & $\begin{array}{l}\text { M: bleeding wounds (Giby, Olany, Polany); stomach ache } \\
\text { (Olany); V: for cows when they piss with blood (Janów } \\
\text { nad Wilią) }\end{array}$ & 3 & 40 \\
\hline Achillea ptarmica L. & czyszcz & $\begin{array}{l}\text { C: cultivated in home gardens (Giby); R: blessed on } \\
\text { Assumption Day (Giby); V: for cows which have just } \\
\text { given birth (Giby) }\end{array}$ & 3 & - \\
\hline Acorus calamus L. & $\begin{array}{l}\text { koławij, francuska } \\
\text { trawa, tatarak }\end{array}$ & $\begin{array}{l}\text { F: bread is baked on its leaves (Giby); M: for hair growth } \\
\text { (Giby) }\end{array}$ & 2 & 20 \\
\hline Aesculus hippocastanum L. & kasztan & M: rheumatism (Olany) & 1 & 20 \\
\hline Agave spp. (?) & oljeander, akjaż & C: cultivated in pots (Giby) & 1 & - \\
\hline Agrostemma githago L. & kąol & $\begin{array}{l}\text { F: stimulant, food seasoning [probably mistaken } \\
\text { information] (Giby) }\end{array}$ & 1 & - \\
\hline Alisma plantago-aquatica L. (?) & babka wodna & M: rabies (Kozły) & 1 & - \\
\hline Allium cepa $\mathrm{L}$. & cebula & $\begin{array}{l}\text { M: ulcerous wounds (Giby); women's genital discharge } \\
\text { (Polany) }\end{array}$ & 3 & 40 \\
\hline Allium sativum $\mathrm{L}$. & czosnek & M: rheumatism (Giby); intestinal parasites (Polany) & 2 & 40 \\
\hline Alnus sp. & olcha & $\begin{array}{l}\text { H: yellow colour dyeing agent (Giby, Narbutowszczyzna); } \\
\text { M: swelling (Giby) }\end{array}$ & 2 & 20 \\
\hline Aloe spp. & aljas & M: wounds (Polany) & 1 & 20 \\
\hline Anchusa officinalis L. & miodunka & M: lung infection (Giby) & 1 & 20 \\
\hline Angelica sp. & dzięgiel & A: hung in the windows to protect against plague (Troki) & 1 & - \\
\hline Antennaria dioica (L.) Gaertn. & no data & $\begin{array}{l}\text { M: folk illness przełamanie (Giby); R: blessed on } \\
\text { Assumption Day (Giby) }\end{array}$ & 2 & 20 \\
\hline Arctium minus (Hill) Bernh. & obrymaczki & M: folk illness fright (Giby); V: swelling in cows (Giby) & 2 & 20 \\
\hline Arctium tomentosum Mill. & rzepnik & M: folk illness róża (Święty Duch) & 1 & 20 \\
\hline $\begin{array}{l}\text { Armoracia rusticana } \\
\text { P. Gaertn., B. Mey. \& Schreb. }\end{array}$ & chrzan & M: cough (Narbutowszczyzna); body weakness (Polany) & 2 & 40 \\
\hline Artemisia abrotanum L. & boże drzewko & C: cultivated in home gardens (Kowno) & 1 & - \\
\hline Artemisia absinthium L. & $\begin{array}{l}\text { piełunek, piołun } \\
\text { (Giby), pałyn } \\
\text { (Narbutowszczyzna), } \\
\text { piołunek (Olany) }\end{array}$ & $\begin{array}{l}\text { M: internal pains, general weakness (Giby); stomach ache } \\
\text { (Narbutowszczyzna), folk illness poruszenie (Olany) }\end{array}$ & 3 & 60 \\
\hline $\begin{array}{l}\text { Artemisia annua } \mathrm{L} \text {. } \\
\text { [A. abrotanum L.] }\end{array}$ & boże drzewko & M: wounds (Polany) & 1 & 20 \\
\hline Artemisia vulgaris L. & bylica & M: general weakness, folk illness fright (Giby) & 2 & 40 \\
\hline Astragalus glycyphyllos L. (?) & bociany, bociani groch & M: intestinal parasites (Giby) & 1 & 20 \\
\hline Atriplex sp., Chenopodium sp. & no data & $\begin{array}{l}\text { H: added to fodder improves egg laying (Janów nad } \\
\text { Wilią) }\end{array}$ & 1 & - \\
\hline Avena sativa $\mathrm{L}$. & owies & $\begin{array}{l}\text { F: ritual food, porridge during fasting periods (Polany); R: } \\
\text { blessed on Assumption Day (Giby) }\end{array}$ & 2 & - \\
\hline Beta vulgaris $\mathrm{L}$. & burok & $\begin{array}{l}\text { F: everyday food, consumed with cabbage and nettles } \\
\text { (Narbutowszczyzna) }\end{array}$ & 1 & - \\
\hline Betula sp. & brzoza & $\begin{array}{l}\text { F: bark as famine food added to bread } \\
\text { (Narbutowszczyzna); H: leaves as dyeing agent } \\
\text { (Narbutowszczyzna); M: kidney infection (Giby); intestinal } \\
\text { problems (Olany) }\end{array}$ & 4 & 40 \\
\hline Brassica oleracea L. & kapusta & $\begin{array}{l}\text { F: everyday food (Narbutowszczyzna); R: blessed on } \\
\text { Assumption Day (Giby) }\end{array}$ & 2 & - \\
\hline Brassica rapa L. & rzepa & F: beverage (Narbutowszczyzna) & 1 & - \\
\hline
\end{tabular}


Table 3 List of plant species used in folk culture of Polish-Lithuanian-Belarusian borderland (Continued)

\begin{tabular}{|c|c|c|c|c|}
\hline Briza media L. & łezki, konopki & M: kidney infection and urine with blood (Oszmiana) & 1 & 20 \\
\hline Bryonia alba L. & przestęp & M: wounds, sore throat (Olany) & 2 & 40 \\
\hline Calendula officinalis $\mathrm{L}$. & $\begin{array}{l}\text { nagietki } \\
\text { (Olany, Polany, Kowno), } \\
\text { nagietek ogrodowy } \\
\text { (Wiłkomierz area) }\end{array}$ & $\begin{array}{l}\text { C: cultivated in home gardens (Kowno); M: against } \\
\text { miscarriage (Olany, Wiłkomierz area); wounds (Wiłkomierz } \\
\text { area); V with oatmeal and wheat : for cattle (Olany); for } \\
\text { cows in the event of an impact, so as not to calve to } \\
\text { early (Polany) }\end{array}$ & 5 & 40 \\
\hline Calluna sp. & wieroz & $\begin{array}{l}\text { F: seeds as famine food, additive to flour } \\
\text { (Narbutowszczyzna) }\end{array}$ & 1 & - \\
\hline Cannabis sativa $\mathrm{L}$. & konopie & M: ulcerous wounds on head (Giby) & 1 & 20 \\
\hline Carum carvi L. & kmin & $\begin{array}{l}\text { F: fruits as seasoning for bread and cheese (Giby); M: } \\
\text { stomach ache, headache (Olany) }\end{array}$ & 3 & 40 \\
\hline $\begin{array}{l}\text { Centaurium pulchellum (Sw.) } \\
\text { Druce }\end{array}$ & centurja & M: folk illness poruszenie (Święty Duch) & 1 & 20 \\
\hline Centaurium sp. & centurja & M: folk illness poruszenie (Polany); liver pain (Grygielany) & 2 & 40 \\
\hline Chelidonium majus L. & złoty groszek & M: eye infection (Giby) & 1 & 20 \\
\hline Chenopodium album $\mathrm{L}$. & lebioda & $\begin{array}{l}\text { F: famine food as cabbage substitute } \\
\text { (Narbutowszczyzna); famine food, eaten boiled with fat } \\
\text { (Giby) }\end{array}$ & 1 & - \\
\hline Chimaphila umbellata (L.) Nutt. & stanawnik & M: folk illness poruszenie (Polany) & 1 & 20 \\
\hline Cichorium intybus L. & uraźnik & M: internal pains, menstrual pains (Giby) & 2 & 40 \\
\hline Cirsium sp/Carduus sp. & oset & $\begin{array}{l}\text { F: everyday food as sorrel substitute, famine food eaten } \\
\text { dried (Narbutowszczyzna) }\end{array}$ & 2 & - \\
\hline Consolida regalis Gray & $\begin{array}{l}\text { regulka (Giby), } \\
\text { rahulka } \\
\text { (Święty Duch) }\end{array}$ & M: colic (Giby, Święty Duch) & 1 & 20 \\
\hline Convallaria sp. & konwalia & $\begin{array}{l}\text { M: internal pains, together with flowers of Cyanus } \\
\text { segetum (Giby) }\end{array}$ & 1 & 20 \\
\hline Convolvulus arvensis $\mathrm{L}$. & powojka & M: general children's weakness, headache (Giby) & 2 & 40 \\
\hline Coriandrum sativum $\mathrm{L}$. & kolendra & F: seasoning for meat (Giby) & 1 & - \\
\hline Cyanus segetum Hill. & $\begin{array}{l}\text { chaber (Giby, } \\
\text { Oszmiana), } \\
\text { wasilki (Graużyszki, } \\
\text { Oszmiana), } \\
\text { bławatki, kwiat } \\
\text { wasilków (Olany) }\end{array}$ & $\begin{array}{l}\text { M: eye infection, colic (Giby); ulcerous wounds } \\
\text { (Graużyszki); cough (Olany); liquid retention in the } \\
\text { organism (Oszmiana) }\end{array}$ & 5 & 100 \\
\hline Cynoglossum officinale L. & repiej & M: rheumatism, wounds (Giby) & 2 & 40 \\
\hline Daucus carota $\mathrm{L}$. & marchew & $\begin{array}{l}\text { M: jaundice, drunk together with Tanacetum vulgare; R: } \\
\text { blessed on Assumption Day (Giby) }\end{array}$ & 2 & 20 \\
\hline Dianthus deltoides $\mathrm{L}$. & iskraczki & M: chest pain (Grygielany) & 1 & 20 \\
\hline Dianthus sp. & $\begin{array}{l}\text { goździk leśny (Giby), } \\
\text { goździk (Kowno) }\end{array}$ & $\begin{array}{l}\text { C: cultivated in home gardens (Kowno); M: intestinal } \\
\text { infection (Giby) }\end{array}$ & 2 & 20 \\
\hline Elsholtzia ciliata (Thunb.) Hyl. & malisa & M: sore throat (Święty Duch) & 1 & 20 \\
\hline Epilobium sp. & podwiejnik & $\begin{array}{l}\text { M: paralysis, in decoction and smoking; common cold } \\
\text { (Oszmiana) }\end{array}$ & 2 & 40 \\
\hline Equisetum arvense L. & skrzyp & $\begin{array}{l}\text { M: kidney infection, decoction with Juniperus communis } \\
\text { frutis, Betula sp. leaves, and two unidentified plants: } \\
\text { borownik świński, koszerka (Giby) }\end{array}$ & 1 & 20 \\
\hline Equisetum pratense Ehrh. & no data & M: internal illnesses in general (Olany) & 1 & 20 \\
\hline Euonymus sp. & $\begin{array}{l}\text { strzmielina, } \\
\text { ćwiekulec }\end{array}$ & $\begin{array}{l}\text { H: material for cobbler's pins (Janów nad Wilią, } \\
\text { Wiłkomierz) }\end{array}$ & 1 & - \\
\hline Ferula sp. & $\begin{array}{l}\text { smrodzieniec, } \\
\text { czarcie łajno }\end{array}$ & B: lighted reveals sorcerer and expels him (Nowogródek) & 1 & - \\
\hline
\end{tabular}


Table 3 List of plant species used in folk culture of Polish-Lithuanian-Belarusian borderland (Continued)

\begin{tabular}{|c|c|c|c|c|}
\hline Fragaria vesca $\mathrm{L}$. & $\begin{array}{l}\text { poziomka (Giby), } \\
\text { paziemacznik } \\
\text { (Grygielany), } \\
\text { poziomnik (Olany) }\end{array}$ & $\begin{array}{l}\text { M: chest pain (Giby, Olany); infertility (Giby); cough } \\
\text { (Grygielany) }\end{array}$ & 3 & 50 \\
\hline Frangula alnus Mill. & no data & M: bladder infection (Święty Duch) & 1 & 20 \\
\hline Geranium palustre L. (?) & ślaz & M: folk illness poruszenie (Olany) & 1 & 20 \\
\hline Glyceria fluitans (L.) R.Br. & manna jadalna & $\begin{array}{l}\text { F: seeds as everyday food, type of gruel (Janów nad } \\
\text { Wilią) }\end{array}$ & 1 & - \\
\hline $\begin{array}{l}\text { Gymnocarpium dryopteris (L.) } \\
\text { Newman }\end{array}$ & urocznik & M: folk illness fright, evil eye (Olany, Oszmiana, Polany) & 2 & 30 \\
\hline $\begin{array}{l}\text { Helianthemum nummularium } \\
\text { (L.) Mill. }\end{array}$ & słonownik & M: kidney infection (Giby) & 1 & 20 \\
\hline $\begin{array}{l}\text { Helichrysum arenarium (L.) } \\
\text { Moench }\end{array}$ & $\begin{array}{l}\text { obrymaczki leśne } \\
\text { (Giby), dramulki } \\
\text { (Grygielany), } \\
\text { miedulki żółte, } \\
\text { nebot (Oszmiana) }\end{array}$ & $\begin{array}{l}\text { C: cultivated in pots [may be a mistaken information]; M: } \\
\text { lung infection (Giby); abdominal pain (Grygielany); } \\
\text { diarrhoea (Oszmiana); R: blessed on Assumption Day } \\
\text { (Giby) }\end{array}$ & 5 & 50 \\
\hline Hordeum vulgare $\mathrm{L}$. & jęczmień & F: ceremonial food, Christmas eve gruel (Polany) & 1 & - \\
\hline Hypericum perforatum $\mathrm{L}$. & świętojańskie ziele & M: folk illness poruszenie, stomach ache (Olany) & 2 & 30 \\
\hline Inula helenium $\mathrm{L}$. & $\begin{array}{l}\text { homian (Janów } \\
\text { nad Wilią, Wiłkomierz) }\end{array}$ & $\begin{array}{l}\text { C: cultivated in home gardens (Janów nad Wilią); M: } \\
\text { scabies (Wiłkomierz); V: cattle's broken legs (Janów nad } \\
\text { Wilią) }\end{array}$ & 3 & 20 \\
\hline Juniperus communis L. & jałowiec & M: respiratory problems, swelling (Polany) & 1 & 40 \\
\hline Knautia arvensis (L.) Coult. & sinowodór & M: folk illness fright in children (Giby) & 1 & 20 \\
\hline $\begin{array}{l}\text { Lagenaria siceraria (Molina) } \\
\text { Standl. (?) }\end{array}$ & tykwa & $\begin{array}{l}\text { C: cultivated in home gardens, } \mathrm{H} \text { : kept on furniture as } \\
\text { adornment (Kowno) }\end{array}$ & 1 & - \\
\hline Lamium album $\mathrm{L}$. & $\begin{array}{l}\text { głucha pokrzywa } \\
\text { (Graużyszki), porzywa } \\
\text { biała (Narbutowszczyzna) }\end{array}$ & $\begin{array}{l}\text { M: heart problems, anaemia (Graużyszki); women's } \\
\text { vaginal discharge (Narbutowszczyzna) }\end{array}$ & 3 & 50 \\
\hline Ledum palustre $\mathrm{L}$. & rozmaryn leśny, bagno & $\begin{array}{l}\text { F: stimulant used as beer adulterant (Wilno area); H: } \\
\text { repellent (Wilno area) }\end{array}$ & 2 & - \\
\hline Leonurus sp. (?) & $\begin{array}{l}\text { serdecznik biały (male), } \\
\text { serdecznik czerwony } \\
\text { (female) [there is a } \\
\text { belief that two } \\
\text { varieties exist : } \\
\text { white - male } \\
\text { and red - female] }\end{array}$ & M: internal infections (Giby) & 1 & 20 \\
\hline Linaria vulgaris Mill. & lonnica & M: smallpox (Graużyszki) & 1 & 20 \\
\hline Linum usitatissimum L. & len & M: swelling, sore throat (Polany) & 2 & 40 \\
\hline Lycopodium clavatum L. & widlak-dziercza & M: wounds (Olany) & 1 & 20 \\
\hline Malus domestica Borkh. & jabłoń & M: heart problems, general weakness (Olany) & 2 & 40 \\
\hline Malva borealis Wallman & ślaz krzaczasty & M: cough (Giby) & 1 & 20 \\
\hline Malva spp. & $\begin{array}{l}\text { ślaz (Giby, Olany), } \\
\text { różyczka } \\
\text { (Janów nad Wilią) }\end{array}$ & $\begin{array}{l}\text { C: cultivated in home gardens (Janów nad Wilią); M: } \\
\text { wounds (Giby); folk illness poruszenie (Olany) }\end{array}$ & 3 & 40 \\
\hline Matricaria chamomilla L. & rumianek & M: stomach ache (Giby) & 1 & 20 \\
\hline Melilotus officinalis (L.) Pall & $\begin{array}{l}\text { borlenin (?) żótty } \\
\text { [the local name is } \\
\text { difficult to decipher] }\end{array}$ & $\begin{array}{l}\text { M: women's problems, } \\
\text { ulcers, swelling (Giby) }\end{array}$ & 3 & 60 \\
\hline Mentha sp. & mięta & $\begin{array}{l}\text { C: cultivated in home gardens (Kowno); } \\
\text { F: refreshing drink (Olany); M: diarrhoea (Polany) }\end{array}$ & 3 & 20 \\
\hline Mentha $\times$ piperita $\mathrm{L}$. & mięta pieprzowa & $\begin{array}{l}\text { M: bone weakness, vomits (Giby); } \\
\text { stomach ache (Olany) }\end{array}$ & 3 & 50 \\
\hline Menyanthes trifoliata $\mathrm{L}$. & bobek & M: ague, stomach problems (Olany) & 2 & 40 \\
\hline
\end{tabular}


Table 3 List of plant species used in folk culture of Polish-Lithuanian-Belarusian borderland (Continued)

\begin{tabular}{|c|c|c|c|c|}
\hline Minuartia sp. (?) & mokrzyca & M: swollen limbs (Giby) & 1 & 20 \\
\hline Nicotiana tabacum L. & tytoń & $\begin{array}{l}\text { M: external parasites; decoction harmful } \\
\text { to lungs, it can cause death. Men sometimes } \\
\text { drink it to avoid being taken to the army (Olany) }\end{array}$ & 2 & 40 \\
\hline Nuphar lutea (L.) Sm. & toteć & $\begin{array}{l}\text { M: "Large leaves of this yellow flower put on a wound, } \\
\text { as they are cold, they extract fire" (Janów nad Wilią) }\end{array}$ & 1 & 20 \\
\hline $\begin{array}{l}\text { Nuphar lutea (L.) Sm. } \\
\text { or Lilium sp. }\end{array}$ & gribilija, żółta lilja & $\begin{array}{l}\text { M: headache, skin infection, folk illness róża, women's } \\
\text { vaginal discharge, decoction together with Lamium } \\
\text { album and Trifolium repens (Giby) }\end{array}$ & 4 & 70 \\
\hline Origanum vulgare L. & lebiodunka & V: medicine for cattle (Giby) & 1 & - \\
\hline Paeonia sp. & piwonja & C: cultivated in home gardens (Kowno) & 1 & - \\
\hline Papaver somniferum L. & $\begin{array}{l}\text { mak (Graużyszki, } \\
\text { Polany), macrek } \\
\text { (Janów nad Wilią) }\end{array}$ & $\begin{array}{l}\text { C: cultivated in home gardens (Janów nad Wilia); } \\
\text { F: an ingredient in the Christmas eve dish called } \\
\text { kucja (Polany); M: toothache (Graużyszki) }\end{array}$ & 3 & 20 \\
\hline Papaver sp. & maczek & C: cultivated in home gardens (Kowno) & 1 & - \\
\hline Parnassia of palustris $\mathrm{L}$. & serdecznik żółty & $\begin{array}{l}\text { M: heart problem (Giby, Święty Duch); external ulcers } \\
\text { (Oszmiana) }\end{array}$ & 2 & 40 \\
\hline $\begin{array}{l}\text { Pelargonium grandiflorum } \\
\text { Willd. }\end{array}$ & juranim & $\begin{array}{l}\text { M: pneumonia, drunk together with milk and honey } \\
\text { (Olany) }\end{array}$ & 1 & 20 \\
\hline Persicaria bistorta (L.) Samp. & wężownik & $\begin{array}{l}\text { F: famine food, grated leaves are bread additives } \\
\text { (Narbutowszczyzna); M: internal pains (Giby) }\end{array}$ & 2 & 20 \\
\hline Phlox paniculata $\mathrm{L}$. & floks & C: cultivated in home gardens (Giby) & 1 & - \\
\hline $\begin{array}{l}\text { Phragmites australis (Cav.) Trin. } \\
\text { ex Steud. }\end{array}$ & trzcina & $\begin{array}{l}\text { A: protects against thunders (Giby); R: blessed on } \\
\text { Assumption Day (Giby) }\end{array}$ & 2 & - \\
\hline Pinus sylvestris $\mathrm{L}$. & sosna & $\begin{array}{l}\text { M: tuberculosis (Polany); lung infection (Grygielany, } \\
\text { Swięty Duch) }\end{array}$ & 2 & 30 \\
\hline Pisum sativum $\mathrm{L}$. & groch & $\begin{array}{l}\text { H: agrarian knowledge about the best periods to sow } \\
\text { the seeds: it says that pea should be sown during the } \\
\text { new moon, so it will have a long flowering period, until } \\
\text { harvest time. If sown with the north wind, it would be } \\
\text { weak, but other people sow it precisely then, because it } \\
\text { prevents pea from vermin. (Janów nad Wilia); V: pea, two } \\
\text { eggs, a live frog and ink as an excellent remedy for cows } \\
\text { which did not moo (Giby) }\end{array}$ & 2 & - \\
\hline Plantago major L. & $\begin{array}{l}\text { babka (Giby), } \\
\text { babka wielka } \\
\text { (Kozły) }\end{array}$ & M: pains, diarrhoea (Giby); wounds (Kozły) & 3 & 60 \\
\hline Plantago media $\mathrm{L}$. & babka średnia & $\begin{array}{l}\text { M: ague (Kozły); pimples, phlegm and short breathing } \\
\text { (Graużyszki) }\end{array}$ & 3 & 60 \\
\hline Plantago sp. & $\begin{array}{l}\text { języczki (Kozły), } \\
\text { babka (Polany, } \\
\text { Święty Duch) }\end{array}$ & M: wounds (Kozły, Polany); diarrhoea (Święty Duch) & 2 & 40 \\
\hline Populus tremula $\mathrm{L}$. & osika & $\mathrm{H}$ : house disinfectant (Olany) & 1 & - \\
\hline Potentilla erecta (L.) Raeusch. & gałgan & M: folk illness poruszenie (Polany, Święty Duch) & 1 & 20 \\
\hline Potentilla sp. & $\begin{array}{l}\text { dzierwanka, } \\
\text { dzierwianka }\end{array}$ & M: lung infection (Olany) & 1 & 20 \\
\hline Primula veris $L$. & kluczyki & M: cough (Olany) & 1 & 20 \\
\hline Prunella vulgaris $\mathrm{L}$. & $\begin{array}{l}\text { brunelka (Giby), } \\
\text { dramulki (Polany), } \\
\text { czemborek } \\
\text { (Graużyszki) }\end{array}$ & $\begin{array}{l}\text { F: refreshing drink, substitute of tobacco (Graużyszki); M: } \\
\text { sore throat (Giby, Polany); headache (Giby) }\end{array}$ & 4 & 40 \\
\hline Prunus cerasus L. & wiśnia & F: additive to lacto-fermented cucumbers (Polany) & 1 & - \\
\hline Prunus padus L. & czeremcha & M: diarrhoea (Polany) & 1 & 20 \\
\hline
\end{tabular}


Table 3 List of plant species used in folk culture of Polish-Lithuanian-Belarusian borderland (Continued)

\begin{tabular}{|c|c|c|c|c|}
\hline Pteridium aquilinum (L.) Kuhn (?) & paprotnik & F: famine food (Narbutowszczyzna) & 1 & - \\
\hline Pulsatilla sp. & sasanka & M: lung problems (Olany) & 1 & 20 \\
\hline Quercus sp. & $d q b$ & M: toothache (Polany) & 1 & 20 \\
\hline Rosa sp. & rajska róża & M: panaceum (Giby) & 1 & 20 \\
\hline Rubus idaeus L. & $\begin{array}{l}\text { malina (Giby), } \\
\text { malinak (Olany) }\end{array}$ & M: common cold (Giby); fever (Olany) & 2 & 40 \\
\hline Rumex sp. & koński szczaw & V: scrofula [horse's illness] (Janów nad Wilią) & 1 & - \\
\hline Ruta graveolens $\mathrm{L}$. & ruta & $\begin{array}{l}\text { C: cultivated in home gardens (Kowno), M: toothache } \\
\text { (Giby) }\end{array}$ & 2 & 20 \\
\hline Salvia officinalis $\mathrm{L}$. & szoławij & M: sore throat (Giby) & 1 & 20 \\
\hline Sambucus nigra L. & bez czarny & M: cough (Grygielany, Narbutowszczyzna) & 1 & 20 \\
\hline Sedum cf acre L. & rozchodnik & M: internal pains (Giby) & 1 & 20 \\
\hline Sedum maximum (L.) Suter & zajęcza kapusta & M: folk illness poddźwignięcie/ochwat (Olany) & 1 & 20 \\
\hline Sempervivum globiferum $\mathrm{L}$. & przeskok & B: used in magic, peasants jump over it (Oszmiana) & 1 & - \\
\hline Sinapis sp. & gorczyca & $\begin{array}{l}\text { M: stomach ache, indigestion (Giby, Polany); "one may } \\
\text { get retarded out of drinking it" (Giby); V: cows should } \\
\text { not eat it, as gorczyca may spoil the milk (Giby) }\end{array}$ & 3 & 40 \\
\hline Sonchus sp. & mleczoj & M: common cold (Giby) & 1 & 20 \\
\hline Sorbus aucuparia L. & jarzębina & M: haemorrhoids (Olany), pain in chest (Polany) & 2 & 40 \\
\hline Succisa pratensis Moench & $\begin{array}{l}\text { macicznik (Giby), } \\
\text { naczniczki (Grygielany), } \\
\text { nizipierśnik (Olany) }\end{array}$ & $\begin{array}{l}\text { M: uterus problems (Giby); somnifacient for children } \\
\text { (Grygielany); ulcers (Olany) }\end{array}$ & 3 & 60 \\
\hline $\begin{array}{l}\text { Symphyotrichum lanceolatum } \\
\text { (Willd.) G.L.Nesom }\end{array}$ & no data & C: cultivated in home gardens (Giby) & 1 & - \\
\hline Syringa vulgaris $\mathrm{L}$. & bez & M: cough (Olany), lung infection (Polany) & 2 & 30 \\
\hline Tagetes spp. (?) & aksamitka, maranta & C: cultivated in home gardens (Kowno) & 1 & - \\
\hline $\begin{array}{l}\text { Tanacetum parthenium (L.) } \\
\text { Sch.Bip. }\end{array}$ & maruna & M: indigestion (Giby) & 1 & 20 \\
\hline Tanacetum vulgare $\mathrm{L}$. & wrotycz & M: jaundice, with carrots [doctrine of signature] (Giby) & 1 & 20 \\
\hline Taraxacum sp. & cykorja polna & $\begin{array}{l}\text { F: everyday food in salads (Lithuania); M: warts } \\
\text { (Lithuania) }\end{array}$ & 2 & 20 \\
\hline Thymus pulegioides $\mathrm{L}$. & $\begin{array}{l}\text { macierzanka (Giby), } \\
\text { czombor (Olany) }\end{array}$ & $\begin{array}{l}\text { M: panaceum (Giby); lung infection (Olany); R: blessed on } \\
\text { Assumption Day (Giby) }\end{array}$ & 3 & 40 \\
\hline Tilia cordata Mill. & lipa & M: cough (Olnay); fever (Polany) & 2 & 40 \\
\hline Tilia sp. & lipa & $\begin{array}{l}\text { F: refreshing drink (Polany); M: common cold (Giby); fever } \\
\text { (Polany) }\end{array}$ & 3 & 40 \\
\hline Trifolium arvense $\mathrm{L}$. & koćki & M: colic (Giby) & 1 & 20 \\
\hline Trifolium aureum Pollich & no data & M: jaundice (Polany) & 1 & 20 \\
\hline Trifolium montanum L. & $\begin{array}{l}\text { grzmotnik, biała } \\
\text { koniczyna, dzięcielina } \\
\text { (biała and wysoka) }\end{array}$ & M: cough (Olany) & 1 & 20 \\
\hline Trifolium pratense L. & koniczyna dzika & F: refreshing drink (Olany); M: internal problems (Olany) & 2 & 20 \\
\hline Trifolium repens $\mathrm{L}$. & $\begin{array}{l}\text { dzięcielinka (Giby), } \\
\text { koniczyna biała } \\
\text { (Graużyszki) }\end{array}$ & $\begin{array}{l}\text { M: women's vaginal discharge, used together with } \\
\text { Nuphar lutea and Lamium album (Giby); herpes } \\
\text { (Graużyszki) }\end{array}$ & 2 & 40 \\
\hline Trigonella foenum-graecum L. & kozieradka & H: to lure wild game (Janów nad Wilią) & 1 & - \\
\hline Tropaeolum majus L. & nasturcja & $\begin{array}{l}\text { M: wounds (Polany); infection, together with Anethum } \\
\text { graveolens and milk (Olany) }\end{array}$ & 2 & 40 \\
\hline Tropaeolum sp. & naśturcie & C: cultivated in home gardens (Giby) & 1 & - \\
\hline Tussilago farfara $\mathrm{L}$. & podbiat & M: headache, folk illness poderwanie (Giby) & 2 & 40 \\
\hline
\end{tabular}


Table 3 List of plant species used in folk culture of Polish-Lithuanian-Belarusian borderland (Continued)

\begin{tabular}{lllll}
\hline Tussilago farfara L. (?) & grzybień & M: cough (Graużyszki) & 1 & 20 \\
Urtica spp. & pokrzywa & F: everyday food with cabbage and beets & 1 & - \\
Urtica urensi L. & (Narbutowszczyzna) & 3 & 60 \\
Vaccinium myrtillus L. & jagodnik & M: paralysis, external ulcers, rheumatism (Graużyszki) & 2 & 20 \\
Vaccinium vitis-idaea L. & brusznicznik & rheumatism (Narbutowszczyzna) & 1 & 20 \\
Valeriana officinalis L. & waleriana majowa & M: rheumatism (Oszmiana) & 2 & 20 \\
Veratrum sp. & ciemiężyca & M: folk illness poderwanie (Giby) & 1 & 20 \\
Verbascum lychnitis L. & no data & M: stomach ache (Grygielany) & 1 & 20 \\
Verbascum sp. & dziewanna & M: healing baths (Giby); chest pain (Narbutowszczyzna); & 3 & 40 \\
Viola arvensis Murray & V: cattle parasites (Giby) & M: headach, lung infection, healing baths for children & 3 \\
Viola tricolor L. & [a name difficult to decipher] & (Giby) & 60 \\
\hline
\end{tabular}

Use categories: $A$ apotropaic, $B$ beliefs, $C$ cultivated in home gardens, $F$ food, $H$ household, $M$ medicinal, $R$ ritual, $V-$ veterinary

domains in the two regions, in historical Lithuania a smaller plant richness was registered in domains such as ritual and, veterinary. Especially, the differences in ritual plant uses are striking: 9 species recorded in this study versus $85 \mathrm{spp}$. in the western Ukraine. In case of food species and plants cultivated in home gardens, more reports were done in the Polish-Lithuanian-Belarusian borderland than in western Ukraine, but the differences are not significant (Fig. 3). However, it should be noted, that the food category is understudied, as some species edible par excellence were recorded only in the medicinal domain: e.g. horseradish (Armoracia rusticana) and sorrel (Rumex spp.) or carrot reported as medicinal and ritual plant. Therefore, we observe a certain bias towards medicinal plants in the whole set of data from the Fischer's study, the researchers' concentration on one domain, which may have influenced the omission of other categories of use, especially those, which were considered quite obvious at that time.

The comparison of information collected in Giby village (71 taxa) - nowadays Poland with the rest of the study area (111 taxa) - nowadays Belarus and Lithuania brought quite unexpected results. The overall similarity between the inner two study region (north-east Poland, and nowadays Belarus and Lithuania) is only 35\%. Remarkably, all the ritual uses of plants (blessed in church ceremonies) were reported only in Giby. Other culturally important species, with at least three different uses in Giby village, not found in Belarus and Lithuania were Achillea ptarmica, Cichorium intybus, Convolvulus arvensis, Melilotus officinalis and Viola arvensis. On the other hand, some common and culturally important plants were only reported in Belarus and Lithuania, namely: Hypericum perforatum, Inula helenium, Juniperus communis, Lamium album, Ledum palustre, Linum usitatissimum, Papaver somniferum, Pinus silvestris, Sambucus nigra, Urtica urens and Vaccinium myrtillus. This analysis leads us to surmise that this data set has its value as a historical material due to the volume of species and the range of an area studied, however the data are too fragmentary to perform any comparison between villages or smaller areas.

The botanical names established by botanists from the Jan Kazimierz University in Lviv are quite reliable. Thirty two taxa were determined to genus level and 121 to species level. However, in the case of nine taxa we put a question mark next to the botanical identification, as we had some doubt about their reliability. These cases are further explained in the Results and Discussion section.

The majority of names are clearly Polish, so we can surmise that the data concerns only Polish-speaking inhabitants. Although Polish names also dominated in the Ukrainian data set, a large number of Ukrainian language names were also recorded [24], in contrast to the present data set which is uniformly Polish.

\section{Medicinal plants}

Plants used in home therapies represent the largest category of uses. We registered 117 taxa and 197 separate uses of plants employed in the treatment of 12 different body systems, of which the most frequently treated were respiratory system disorders (40 plant uses), followed by digestive (33 plant uses) and skin problems (31 uses). A considerable number of plant uses were found in the sub-category of folk illnesses (22 uses). The folk illnesses included evil eye (uroki), fright (przestrach), róża (a skin illness), poderwanie (a muscular problem from lifting heavy weights) and poruszenie (a digestive problem). They were classified as folk illnesses, as they had no strict equivalent in the biomedical classification of diseases, and normally their treatment also included some 


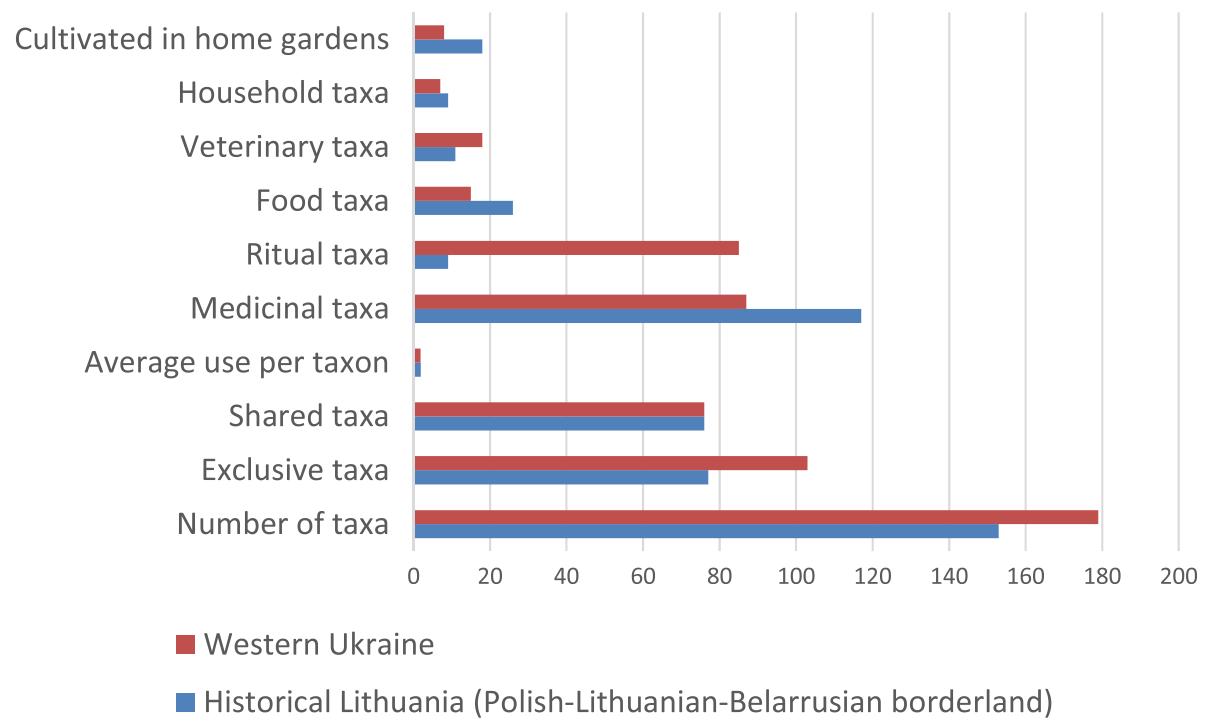

Fig. 3 The comparison of taxa and category of uses between Polish-Lithuanian-Belarusian borderland and western Ukraine

magical action apart from using medicinally useful plants. The most frequently treated illnesses in the study region, according to the number of taxa, were: wounds including ulcers and boils (18 different taxa), coughs (10), chest pain (8), stomach ache (8) and headache (6). The species with the highest RI value (the most versatile plants) were: Cyanus segetum (100), Nuphar lutea (70), Artemisia absinthium (60), Melilotus officinalis (idem), Plantago major, Plantago media, Succisa pratensis, Urtica urens, Viola arvensis. Moszyński describes in his work Eastern Polesia [12], Nuphar lutea as a very visible plant in the Polesia landscape and that rhizomes of this species were used in folk treatment, while seeds were eaten by locals.

Medicinal plants were used with greater frequency internally (89 uses) than in the form of external applications (66). We had no data about 42 plant uses, in this respect. The great majority of plants were used solely, and herbal mixtures were reported very rarely. They were only mentioned in the case of remedies against vaginal discharge (Nuphar lutea, Lamium album, Trifolium repens), and jaundice (Tanacetum vulgare, Daucus carota) - this is also an example of the doctrine of signatures. Infections were treated with Tropaeolum majus flowers, seeds of Anethum graveolens and milk. Similarly, chest problems were cured with Pelargonium grandiflorum, milk and honey. Contemporary studies also confirm that respiratory problems have traditionally been treated with "sweet" remedies [33, 34]. Characteristically, no pharmaceuticals were combined with plant medicines, or at least the field workers did not report this practice.

When we compared medicinal plant species used in this region and western Ukraine (87 taxa) in the 1930s, we found a greater species richness in the PolishLithuanian-Belarusian borderland (117 taxa). Only 45 plant species were shared between regions, and the similarity in plant composition reached $44 \%$. The species with the highest RI value were different too, and only Artemisia absinthium was shared between the regions. Other most versatile species in western Ukraine were Achillea millefolium (100), Tussilago farfara (100), Veratrum album (87.5), Allium sativum (75) and Viola tricolor L. (75). Species important in folk medicine in western Ukraine, such as Matricaria chamomilla, was recorded with single uses in phytotherapy in historical Lithuania and other important medicinal plants, such as Arnica montana were not reported in this study. Many more similarities were found in body systems and illnesses treated with plants, as well as in modes of use and the prevalence of single plant remedies over herbal mixtures [24]. All in all, although peasants in the compared regions used quite different species to treat illnesses, they were used in similar ways, and to treat a similar spectrum of illnesses.

\section{Other uses of plants \\ Food}

This category embraces 26 taxa (29 records). Plants were used in a few subcategories: everyday food (9 records), famine food (7 records), beverages ( 5 records), spices (3 records), ceremonial food (3 records) and stimulants (2 records). The everyday food subcategory embraces plants used for different purposes. Bread was baked on sweet flag (Acorus calamus), and the seeds of another aquatic plant, Glyceria fluitan, were collected to make gruel. Cherry leaves were added to pickled cucumbers, and nettle 
(Urtica dioica) was eaten with cabbage (Brassica oleracea) and beet (Beta maritima). In one of the villages, as a substitute for sorrel (Rumex spp.), a wild plant called oset (Cirsium sp. or Carduus sp.) was used. There was one unknown plant called, locally, legiesie, which was prepared as lactofermented food. Famine plants are represented by six species. They were used during "war time", which may be ascribed to World War I or/and the Polish-Soviet war in 1920. These were oset (Carduus sp. or Cirsium sp.), paprotnik (Pteridium aquilinum?) and bistort (Persicaria bistorta). Lamb's quarters (Chenopodium album), common heather (Calluna vulgaris - seeds ground and added to flour) and even small pieces of birch wood (Betula sp.) were used during food shortages. Five plants were used as herbal teas: mint (Mentha sp.), lime flowers (Tilia sp.) but also common self-heal (Prunella vulgaris), red clover (Trifolium pretense) and finally turnip leaves (Brassica rapa), which were used in dried form (collected during the spring time) for a drink called chtodniczek. In the subcategory of ceremonial food there are only three plants: two used during the Christmas Eve supper: barley (Hordeum vulgare, in the form of gruel) and poppy (Papaver somniferum, an ingredient for the special Christmas dish kutia), and one used during fasting - oats (Avena sativa, porridge). Two plants were considered stimulants: common self-heal (Prunella vulgaris), whose leaves were smoked, and marsh Labrador tea (Ledum palustre), used to adulterate low alcohol beer.

The wild food plant uses in the studied Fischer's archives contain species already reported from Eastern Europe as wild edible plants used by local population. Some of the rarest uses and those of largest interest is the alimentary use Pteridium aquilinum and Persiacria bistorta, only documented by Fedorowski [16].

The most intriguing plant in the subcategory of herbal teas is turnip (Brassica rapa). We do not know how the tea was used, but the local name of turnip tea chtodniczek (means: cooler), suggests its designation. It is the first-noted use of turnip as a beverage plant in this part of Europe [35]. Baking bread on plant leaves was intended to prevent burnt flour from sticking to the fresh bread. Many different species were traditionally used for this purpose - cabbage, oak, maple and sweet flag [36]. This baking additive is still present in the Podlasie region in NE Poland [37]. Famine plants were widely used in Poland, all the listed plants had been noted before [38, 39]. The local name kakol (meaning common corn-cockle) on one of the filecards, with the description "for seasoning food" is probably misleading. Agrostemma githago, commonly called kakol, was a wellknown toxic plant and a common weed, hence its possible use as a spice is improbable - perhaps a narcotic use should be considered, or maybe some other species was involved.

\section{Household}

The 'Household' category is represented by 9 taxa (15 use reports), and could be distributed into the following subcategories: dyeing (7), animal wellbeing (2), repellents (2), shoemaking (2), hunting (1) and agricultural knowledge (1). Plant-derived dyeing agents were as follows: blueberry (Vaccinium myrtillus) for black, with mud also used in the process. Yellow was made by using alder bark or mature "cones" (Alnus sp.), as well as birch leaves (Betula sp.), with alum as a mordant. The case of a plant called miczan, a black dyeing agent, remains unresolved due to the lack of a Latin name in the original data set. Dyeing fabrics with plants has a long history, and it is worthy of note that even during the industrial era, plant dyeing agents were still in use in Poland and adjacent areas [40]. Btoto (mud) used in the dyeing process could mean copper sulfate [41]. Another mordant - alum used with birch leaves, was also common [41].

Repellents were prepared out of Ledum palustre, which protected cereals in the granary, and aspen bark (Populus tremula), mixed with rye flour, was used for house disinfection, removing parasites. Ledum palustre, easily available in the region, and used as a repellent, is still in use in NE Poland [37]. Using a mixture made of rye flower and aspen bark against parasites was practically unknown elsewhere, and presumably ineffective [42]. Probably there was some kind of knowledge or superstition about one or both of these plants behind this idea of parasite management, but unfortunately we do not have any data to compare it with.

Interesting information about fenugreek (Trigonella foenum-graecum) was found in the data set - it was used by hunters "to lure game animals". In Polish ethnobotanical materials hunting is not a common category. The usage of fenugreek mentioned on Fischer's filecards could mean adding spice to attract wild animals by preparing and leaving it in the forest in special food "balls". People in Augustów Forest (close to Suwałki) still leave food for wild boar in one specific place as a hunting lure, despite the fact that it is illegal nowadays (unpublished materials, P.K.). One piece of information concerns local knowledge about pea growing. It says that pea (Pisum sativum) should be sown during the new moon, so it will have a long flowering period, until harvest time. If sown with the north wind, it would be weak, but other people sow it precisely then, because it prevents pea from vermin.

\section{Ritual}

Fischer's archives contain very few records concerning ritual plants from the study area: only 9 species of plants are mentioned as traditionally blessed on Assumption Day (August, $15^{\text {th }}$ ) in Giby near Suwałki, and the painting of Easter eggs with onion is recorded in two 
localities. Maybe these traditions were too obvious for the informants, who did not pay enough attention to them. On the other hand the tradition of blessing herbs on Assumption, very rich in southern Poland, involved only a few species in NE Poland (compare [36, 37] with $[43,44]$ for southern Poland). Within the same data set from western Ukraine, 85 different species were used in the rituals by peasants from the region [24]. A similarly characteristic division between NE Poland and the rest of the country was also found on a map prepared by Moszyński and published in 1935, which presented plants stuck into thatched roofs, walls and the like on Midsummer day (23 $3^{\text {rd }}$ of June). NE Poland (today Lithuania) was characterized by the prevalence of use of aspen, nettle and juniper, whose apotropaic powers could be easily explained - their aim was to do harm to witches, who were believed to be particularly active during Midsummer night. In the rest of the country more numerous species were used, with more obscure apotropaic genesis, such as burdock, mugwort, wormwood and lime tree $[45,46]$.

\section{Veterinary}

Within this category 11 different species were recorded. Achillea millefolium, Arctium minus, Calendula officinalis, Inula helenium, Origanum vulgare, and Sinapis sp. were the species used especially in the treatment of cows. One recipe from Giby village mentioned pea (Pisum sativum), two eggs, a live frog and ink as an excellent remedy for cows which did not moo. Sorrel (Rumex sp.) was used in the treatment of one particular illness which affected horses, namely scrofula. The afore-mentioned plants had a purely curative character and we did not notice anything like the continuity between fodder and veterinary plants used for animal wellbeing, which was observed in the data set from western Ukraine [24].

\section{Plants cultivated in home gardens}

Home garden plants are represented by 18 species, mainly ornamental: peony (Peonia sp.), marigold (Calendula officinalis), Tagetes spp., mallow (Malva sp.), garden nasturtium (Tropaeolum majus), perennial phlox (Phlox paniculata), panicled aster (Symphyotrichum lanceolatum) and sneezewort (Achillea ptarmica). Two species were mentioned as potted plants - agave (Agave sp.) and, surprisingly - dwarf everlast (Helichrysum arenarium). Gourd (Lagenaria siceraria) was planted for its fruits, which then were used indoors - mounted on wardrobes and tile stoves for ornamental purposes. This group embraces valuable and useful plants, which could simultaneously be treated as ornamental and medicinal: mint (Mentha spp.), rue (Ruta graveolens), poppy
(Papaver sp.), elecampane (Inula helenium) and southernwood (Artemisia abrotanum).

In the light of the abundance of plant species described from historical Lithuania in the $18^{\text {th }}$ and $19^{\text {th }}$ centuries as common garden plants [11, 47], Fischer's list is rather short and must have been collected randomly. Mentioning dwarf everlast (Helichrysum arenarium) as a potted plant seems to be a mistake. It is a native and common plant in Lithuania and Belarus, requiring well-drained soil with sand and gravel, typical in a postglacial landscape. There is no need to plant it in a pot, as it is easily accessible in the wild or can be cultivated in the garden. The fruits of gourd, Polish tykwa, were presumably Lagenaria siceraria fruits used for interior decoration. Wyżycki [11] described how to process gourd, to preserve it for long time, which suggests that its use was common long before Fischer's research.

\section{Conclusions}

The presented ethnobotanical data constitute a valuable contribution to the ethnobotany of Eastern Europe as a whole, however due to their versatility of information they are less likely to lead to a conclusive synthesis. The presented list of plants and the reference list may however be rich sources for future studies of the ethnobotany of the Polish diaspora in Lithuania and diachronic studies in north-east Poland and Belarus.

\section{Abbreviations}

PTL: Polskie Towarzystwo Ludoznawcze (Polish Ethnological Society)

\section{Acknowledgements}

The authors are grateful to Professor Gustaw Juzala-Departi for useful prompts and references.

\section{Funding}

The research was financed by funds from the National Science Centre, Poland [2014/13/D/HS3/03697].

\section{Availability of data and material}

The data set analysed during the current study is available in Archives of the Polish Ethnological Society (Polskie Towarzystwo Ludoznawcze), F. JoliotCurie 12, 50-383 Wrocław, Poland; website: ptl.info.pl

\section{Authors' contributions}

The majority of work on all stages of research (digitalization of archives, entering data in the database and writing of the manuscript) was performed by MK. $Ł$ initiated the study and supervised the analysis of local plant names and the accuracy of the botanical name identification. He also performed a critical check of the literature and contributed to the final version of the manuscript. PK participated in data analysis, checked Latin names, prepared Fig. 1, contributed useful references and participated in writing the manuscript. All authors read and approved the final manuscript.

Competing interests

The authors declare that they have no competing interests.

Consent for publication

Not applicable

Ethics approval and consent to participate Not applicable 


\section{Publisher's Note}

Springer Nature remains neutral with regard to jurisdictional claims in published maps and institutional affiliations.

\author{
Author details \\ 'Institute of Ethnology and Cultural Anthropology, University of Łódź, \\ Lindleya 3/5, 90-131 Łódź, Poland. 'Institute of Botany, Jagiellonian \\ University, Kopernika 27, 31-501 Kraków, Poland. ${ }^{3}$ Department of Botany, \\ Institute of Biotechnology, University of Rzeszów, Werynia 502, 36-100 \\ Kolbuszowa, Poland.
}

\section{Received: 23 January 2017 Accepted: 7 March 2017}

Published online: 23 March 2017

\section{References}

1. Davies N. Europe: A history. London: Random House; 1997.

2. Petkeviciute Z, Savickiene N, Savickas A, Bernatoniene J, Simaitiene Z, Kalveniene Z, Lazauskas R, Mekas TA. Urban ethnobotany study in Samogitia region, Lithuania. J Med Plant Res. 2010;4(1):064-71.

3. Engelking A, Golachowska E, Zielińska A (eds.). Tożsamość, Język, Rodzina. Z badań na pograniczu słowiańsko-bałtyckim. Warszawa: Slawistyczny Ośrodek Wydawniczy; 2008

4. Šeškauskaitè D, Gliwa B. Rūtà, die Nationalblume der Litauer: Zur Kulturgeschichte der Weinraute (Ruta graveolens L.) und zur Etymologie von litauisch rūtà und deutsch Raute. Anthropos. 2002;97(2):455-67.

5. Šeškauskaitè D, Gliwa B. Some Lithuanian ethnobotanical taxa: a linguistic view on Thorn Apple and related plants. J Ethnobiol Ethnomed. 2006;2(1):1.

6. Seskauskaite D, Gliwa B. The Botanical Identity and Cultural Significance of Lithuanian Jovaras: An Ethnobotanical Riddle. In: Ethnobotany in the new Europe: people, health, and wild plant resources. New York - Oxford: Berghahn Books; 2010. p. 246-62.

7. Petkevičius R, Typek J, Bilek M. Jan Kazimierz Muszyński (1884- 1957) prekursorem badań etnobotanicznych na Litwie. Etnobiologia Polska. 2014;4:55-82.

8. Balys J. Liaudies magija ir medicina [Folk magic and folk medicine]. Bloomington: Author; 1951.

9. Muszyński J. Wileńskie zioła ludowe. Wiadomości Farmaceutyczne 1927;2122:469-476.

10. Syreński S. Zielnik Herbarzem z ięzyka Łacinskiego zowia To iest Opisanie własne imion, kształtu, przyrodzenia, skutkow, y mocy Zioł wszelakich Drzew Krzewin y korzenia ich, Kwiatu, Owocow, Sokow Miasg, Zywic y korzenia do potraw zaprawowania Takze Trunkow, Syropow, Wodek Lekiwarzow Konfektow [...]. Kraków: W Drukarni Bazylego Skalskiego; 1613.

11. Wyżycki GJ. Zielnik ekonomiczno-techniczny: czyli opisanie drzew, krzewów i roślin dziko rosnących w kraju, jako też przyswojonych, z pokazaniem użytku ich w ekonomice, rękodziełach, fabrykach i medycynie domowej, z wyszczególnieniem jadowitych i szkodliwych [...] ułożony dla gospodarzy i gospodyń. Vol. 2. Wilno: author's edition; 1845

12. Moszyński K. Polesie wschodnie. Materiały etnograficzne z wschodniej części byłego powiatu mozyrskiego oraz powiatu rzeczyckiego. Wydawnictwo Kasy im. Mianowskiego: Warszawa; 1928.

13. Orzeszkowa E. Kwiaty i ludzie nad Niemnem. Wisła: miesięcznik geograficzno-etnograficzny. 1888;2:675-703.

14. Orzeszkowa E. Kwiaty i ludzie nad Niemnem. Hommes et plantes des bords du Niemen. Wisła: miesięcznik geograficzno-etnograficzny. 1891;5:235-47.

15. Wieras Z. [Верас 3]. Беларуска-польска-расейска-лацінскі батанічны слоўнік. Wilno: Drukiem S. Bekiera; 1924

16. Fedorowski M. Lud białoruski na Rusi Litewskiej: materyały do etnografii słowiańskiej zgromadzone w latach 1877-1891. T. 1. Kraków: Akademia Umiejętności; 1897.

17. Graniszewska M, Leśniewska H, Mankiewicz-Malinowska A, Galera H. Rośliny użyteczne... Michała Fedorowskiego - dzieło odnalezione po 130 latach. Etnobiologia Polska. 2013;3:63-120.

18. Łuczaj Ł, Köhler P, Pirożnikow E, Graniszewska M, Pieroni A, Gervasi T. Wild edible plants of Belarus: from Rostafiński's questionnaire of 1883 to the present. J Ethnobiol Ethnomed. 2013:9:1.

19. Sõukand R, Hrynevich $Y$, Vasilyeva I, Prakofjewa J, Vnukovich $Y$, Paciupa J, Hlushko A, Knureva Y, Litvinava Y, Vyskvarka S, Silivonchyk H, Paulava A, Kõiva M, Kalle R. Multi-functionality of the few: current and past uses of wild plants for food and healing in Liubań region, Belarus. J Ethnobiol Ethnomed. 2017;13:10
20. Czekanowski J. Półwiecze Towarzystwa Ludoznawczego. Lud. 1946;36:33-88.

21. Jasiewicz Z, Rieszetow AM. Korespondencja między Adamem Fischerem a Dimitrem Konstantynowiczem Zieleninem. Etnografia Polska. 2003; 47(1-2):31-47.

22. Suchecka P. Korespondencja Adama Fischera w Zbiorach Archiwum Naukowego Polskiego Towarzystwa Ludoznawczego. In: Jasiewicz Z, Karwicka T, editors. Przeszłość etnologii polskiej w jej teraźniejszości. Poznań: Dalet; 2001. p. 71-8.

23. Gołąbek J. I Zjazd Filologów Słowiańskich w Pradze. Przegląd Pedagogiczny. 1929;33(13):793-4.

24. Kujawska M, Łuczaj Ł, Typek J. Fischer's Lexicon of Slavic beliefs and customs: a previously unknown contribution to the ethnobotany of Ukraine and Poland. J Ethnobiol Ethnomed. 2015;11:85.

25. Fischer A. Rośliny w wierzeniach i zwyczajach ludu polskiego. Lud. 1929:28:240-7.

26. Fischer A. Rośliny w wierzeniach i zwyczajach ludu polskiego. Kwestionariusz. Orli Lot. 1930:5-6:85-86.

27. Łowmiański H. Studia nad dziejami Wielkiego Księstwa Litewskiego. Poznań: Uniwersytet im. Adama Mickiewicza; 1983.

28. Dąbrowki G. Prosta etnografia Wileńszczyzny. Wrocław: Katedra Etnologii i Antropologii Kulturowej UWr; 2013.

29. Łaguna S. Teki etnograficzne[manuscript nr 4439]. Wrocław: Ossolinum; no date.

30. The Plant List. 2013. http://www.theplantlist.org/. Accessed 06 Jan 2017.

31. Prance GT, Balée W, Boom BM, Carneiro RL. Quantitative ethnobotany and the case for conservation in Amazonia. Conserv Biol. 1987;1(4):296-310.

32. Bennett BC, Prance GT. Introduced plants in the indigenous pharmacopeia of northern South America. Econ Bot. 2000;54:90-102.

33. Paluch A. Świat roślin w tradycyjnych praktykach leczniczych wsi polskiej. Wrocław: Wydawnictwo Uniwersytetu Wrocławskiego; 1984.

34. Zamudio F, Kujawska M, Hilgert NI. Honey as Medicinal and Food Resource. Comparison between Polish and Multiethnic Settlements of the Atlantic Forest, Misiones, Argentina. Open Complement Med J Special Issue Med Ethnobiol. 2010;2:58-73.

35. Sõukand R, Quave CL, Pieroni A, Pardo-de-Santayana M, Tardío J, Kalle R, Łuczaj Ł, Svanberg I, Kolosova V, Aceituno-Mata L, Menendez-Baceta G. Plants used for making recreational tea in Europe: a review based on specific research sites. J Ethnobiol Ethnomed. 2013;9:58.

36. Gaweł A. Zwyczaje, obrzędy i wierzenia agrarne na Białostocczyźnie od połowy XIX do poczatku XXI wieku. Białystok: Księgarnia Akademicka; 2009.

37. Klepacki P. Rośliny użytkowe w Puszczy Knyszyńskiej i Beskidzie Niskim. Etnobiologia Polska. 2016;6:31-116.

38. Łuczaj Ł, Szymański W. Wild vascular plants gathered for consumption in the Polish countryside: a review. J Ethnobiol Ethnomed. 2007;3:17.

39. Łuczaj Ł. Dziko rosnące rośliny jadalne użytkowane w Polsce od połowy XIX w. do czasów współczesnych. Etnobiologia Polska. 2011;1:57-125.

40. Kowecka E. Farbiarstwo tekstylne na ziemiach polskich (1750-1870). Stud i Mat z Hist Kult Mat. 1963;12(2):1-183.

41. Mowszowicz J. Przewodnik do oznaczania krajowych roślin zielarskich. Warszawa: Państwowe Wydawnictwo Rolnicze i Leśne; 1985.

42. Sõukand R, Kalle R, Svanberg I. Uninvited guests: traditional insect repellents in Estonia used against the clothes moth Tineola bisselliella, human flea Pulex irritans and bedbug Cimex lectularis. Insect Sci. 2010;10:150.

43. Łuczaj $Ł$. Changes in Assumption Day Herbal Bouquets in Poland: a nineteenth century study revisited. Econ Bot. 2011;65(1):66-75.

44. Łuczaj Ł. Rośliny święcone w bukietach w dniu Matki Boskiej Zielnej w cerkwiach prawosławnych na przedpolu Puszczy Białowieskiej. Plants in bouquets blessed on assumption day in orthodox churches in the vicinity of the Białowieża forest. Etnobiologia Polska. 2013;3:55-62.

45. Moszyński K. Atlas kultury ludowej w Polsce, vol. 2. Kraków: Polska Akademia Umiejętności; 1935.

46. Kujawska M. Folk biology of Slavic-speaking peoples. Kazimierz Moszyński and his comparative studies. In: Svanberg I, Łuczaj $Ł$, editors. Pioneers in European Ethnobiology. Uppsala: Uppsala Studies in Eastern Europe 4; 2014 p. 181-199.

47. Jundziłł B. Opisanie roślin w Prowincyi Wielkiego Xięstwa Litewskiego naturalnie rosnących według układu Linneusza. Wilno: Drukarnia Pijarów; 1791. 